\title{
WEAK UNIQUENESS SETS FOR DISCRETE GROUPS
}

\author{
BY
}

\author{
MAREK BOŻEJKO AND TADEUSZ PYTLIK
}

\begin{abstract}
ABSTRACr. For discrete groups we introduce a new class of sets, called weak uniqueness sets, which for abelian groups contains the class of sets of uniqueness. Considered is the problem of determining groups for which every finite set is a weak uniqueness set. Some examples are given.
\end{abstract}

1. Introduction. Investigations of B. Riemann, G. Cantor and de la ValléePoussin of the convergence types of trigonometric series have led, among others, to the concept of sets of uniqueness. A subset $E$ of $[0,2 \pi)$ is called a set of uniqueness if each trigonomeric series convergent to 0 outside $E$ equals 0 identically (cf. [20, IX, §6]). These sets were studied by several authors, e.g. Loomis and Meyer have extended the concept from the group of integers to any locally compact abelian group. It is known that finite, countable compact, and some perfect sets are sets of uniqueness, but their full description is still to be done. Most papers deal with more and more subtle examples. For more information consider N. Bary [1] or Y. Meyer [12].

In the present paper we introduce the concept of a weak uniqueness set and prove some natural properties of the class of such sets. For instance, it contains the class of sets of uniqueness. Moreover, the concept of a weak uniqueness set can be simply extended to nonabelian groups, but in that case its study becomes difficult, as it depends essentially on the group structure as well as on the structure of the maximal ideal space of the group algebra.

Let $G$ be a discrete group and $\Re(G)$ the space of all maximal two-sided ideals in $l^{1}(G)$. A subset $E$ of $\Re(G)$ will be called a weak uniqueness set if the ideal equal to the common part of all ideals of $E$ is a weakly* dense subset of $l^{1}(G)$.

We concentrate upon the question when one-element sets are weak uniqueness ones. Some partial, positive and negative, results are presented below and for the groups of type FC we succeed in obtaining a full characterization. At the end of the paper there are three examples which show that, among others, the intuitions related to weak uniqueness sets in the abelian case do not hold, in general, in the nonabelian case.

Received by the editors March 4, 1977.

AMS (MOS) subject classifications (1970). Primary 22D15, 42A48, 43A20; Secondary 22D20, 43A45.

Key words and phrases. Weak uniqueness sets, weak* topology, maximal ideal space. 
2. Density in the weak* topology. We start with an elementary but useful

Proposition 2.1. Let $X$ be a Banach space and let $Y$ be a linear subspace in $X^{*}$. The subspace $Y$ is weakly* dense in $X^{*}$ if and only if $Y$ is a total set for $X$ (i.e., $Y$ separates points of $X$ ).

This proposition is well known; see, e.g., Exercise 41, §V.7 (page 439) of Dunford and Schwartz, Linear operators, Part I, Interscience, 1958.

Some remarks concerning notations and terminology are now in order. Let $H$ be an infinite set. We will denote by $l^{1}(H), l^{\infty}(H)$ and $c_{0}(H)$ the Banach spaces consisting of all absolutely summable functions on $H$, all bounded functions on $H$, and all functions on $H$ vanishing at infinity (the closure in $l^{\infty}(H)$ of functions with finite supports), respectively. For $f$ in $l^{1}(H)$ and $\varphi$ in $l^{\infty}(H)$ (or $\varphi$ in $c_{0}(H)$ ) the scalar product $\langle f, \varphi\rangle$ will be defined by the formula

$$
\langle f, \varphi\rangle=\sum_{x \in H} f(x) \overline{\varphi(x)} .
$$

This scalar product forces a unique sense of identifications $c_{0}(H)^{*}=l^{1}(H)$, $l^{1}(H)^{*}=l^{\infty}(H)$, and thus the weak and weak* topologies in $l^{1}(H)$ are well defined. For a subset $I$ of $l^{1}(H)$ the annihilator An $I$ of $I$ in $l^{\infty}(H)$ will be the subspace

$$
\text { An } I=\left\{\varphi \in l^{\infty}(H):\langle f, \varphi\rangle=0 \text { for all } f \in I\right\} .
$$

Proposition $2.2 \mathrm{~A}$ linear subspace $I \subset l^{1}(H)$ is weakly* dense in $l^{1}(H)$ if and only if An $I \cap c_{0}(H)=\{0\}$.

Proof. By Proposition 2.1 it follows that $I$ is weakly* dense in $l^{1}(H)$ if and only if for every nonzero $\varphi \in c_{0}(H)$ there exists an $f$ in $I$ such that $\langle f, \varphi\rangle \neq$ 0 .

THEOREM 2.3. Let $G$ be an infinite group and let I be a left ideal in $l^{1}(G)$. If I has finite codimension, then it is weakly* dense in $l^{1}(G)$.

Proof. Since An $I$ is a finite dimensional left-invariant subspace in $l^{\infty}(G)$, it consists of left almost periodic functions (a bounded function on $G$ is left almost periodic if the set of all its left translations is a precompact set in $\left.l^{\infty}(G)\right)$ and since the only left almost periodic function in $c_{0}(G)$ is the zero function, ${ }^{1}$ we get An $I \cap c_{0}(G)=\{0\}$. Thus, by Proposition 2.2, our theorem follows.

Remarks. (1) Theorem 2.3 is not true for subspaces of finite codimension.

(2) For every group $G$ the algebra $l^{1}(G)$ contains a two-sided ideal of finite

1 Let $\varphi$ be a nonzero function in $c_{0}(G)$ and let $K$ be a finite subset of $G$ such that $|\varphi(x)| \leqslant \frac{1}{3}\|\varphi\|$ outside of $K$. If we choose inductively a sequence $x_{1}, x_{2}, \ldots$ of elements in $G$ such that $x_{n} \notin\left\{x_{1}, x_{2}, \ldots, x_{n-1}\right\} K K^{-1}$, then $x_{i} K \cap x_{j} K=\varnothing$ for all $i \neq j$ and thus $\left\|\varphi_{x_{i}}-\varphi_{x_{j}}\right\|>\frac{1}{3}\|\varphi\|>0$. The sequence $\varphi_{x_{1}}, \varphi_{x_{2}}, \ldots$ has no convergent subsequences. 
codimension, for example, the ideal of all functions $f$ for which $\sum_{x \in G} f(x)=$ 0 .

Let $G$ be a discrete group and let $\Re(G)$ denote the set of all two-sided maximal ideals in $l^{1}(G)$. For a subset $E$ in $\Re(G)$ let $k(E)=\bigcap_{M \in E} M$ be . the kernel of $E$, and for a subset $I$ in $l^{1}(G)$ let $h(I)=\{M \in \mathscr{N}(G)$ : $M \supseteq I\}$ be the hull of $I$. Then there exists a unique topology in $\Re(G)$, called the Jacobson topology (see [2, 3.1.1]), for which $E \rightarrow \bar{E}=h k(E)$ is the closure operation. In this topology $\Re(G)$ is a quasicompact $T_{1}$-space [2, 3.1.4 and 3.1.8].

A closed subset $E \subset \mathfrak{N}(G)$ will be called a weak uniqueness set if $k(E)$ is a weak* dense subset in $l^{1}(G)$.

3. Connections with the classical definition. Consider the case when $G$ is a discrete abelian group. The classical definition [12] says that a closed subset $E$ of $G$ is a set of uniqueness if the only pseudomeasure $T$ carried by $E$ whose Fourier transform vanishes at infinity is zero. An equivalent condition is: If a function $\varphi$ belongs to $c_{0}(G)$ and has spectrum in $E$, then $\varphi$ is zero.

Let us recall that $\varphi$ has spectrum in $E$ if $\langle f, \varphi\rangle=0$ for every function $f$ from the ideal $I_{0}(E)$, the smallest closed ideal in $l^{1}(G)$ whose hull is $E$ (it is the closure in $l^{1}(G)$ of the set of those functions whose Fourier transform vanishes in a neighbourhood of $E$ ). Therefore $E$ is a set of uniqueness if and only if $I_{0}(E)$ is weakly* dense in $l^{1}(G)$.

One can observe that the classical definition and the definition of weak uniqueness sets look similar although a priori the first one is stronger than the second. In fact they coincide for sets with spectral synthesis, because then $I_{0}(E)=h(E)$. To see that they are nonequivalent we should find a compact set $E$ (without spectral synthesis) for which the ideal $h(E)$ is weakly* dense in $l^{1}(G)$ but $I_{0}(E)$ is not. A construction of such a set for the group of integers was given by $\mathrm{T}$. W. Körner in [11]. ${ }^{2}$

4. Some general results. In this section we shall show that the class of weak uniqueness sets has two natural properties. First, the union of two weak uniqueness sets is also of this type, and second, each weak uniqueness set is small in the sense that it has empty interior.

LEMMA 4.1. If two two-sided ideals $I_{1}, I_{2}$ are weakly* dense in $l^{1}(G)$, then so is the ideal $I_{1} \cap I_{2}$.

${ }^{2}$ Let $G$ be a discrete abelian group and let $E$ be a Helson set in $G$. The annihilator An $h(E)$ of the ideal $h(E)$ is equal to the set of all Fourier transforms of measures supported by $E$. By the Helson Theorem [5, 41.18.d], An $h(E) \cap c_{0}(G)=\{0\}$. Thus Helson sets are weak uniqueness sets.

Körner gave an example of a Helson set in the circle which carries a nonzero pseudo-function. Thus $E$ is not a set of uniqueness. 
Proof. Note first that a two-sided ideal $I$ is weakly* dense in $l^{1}(G)$ if and only if for every nonzero function $\varphi \in c_{0}(G)$ there exists $f \in I$ such that $f * \varphi \neq 0$. Now take a nonzero function $\varphi \in c_{0}(G)$. Since $I_{1}$ is weakly* dense in $l^{1}(G)$, there exists $f_{1}$ in $I_{1}$ such that $f_{1} * \varphi \neq 0$. By the same argument there exists $f_{2}$ in $I_{2}$ such that $f_{2} *\left(f_{1} * \varphi\right) \neq 0$ (note that $f_{1} * \varphi \in c_{0}(G)$ ). Since $f_{2} * f_{1}$ belongs to $I_{1} \cap I_{2}$, our lemma follows.

THEOREM 4.2. The union of two weak uniqueness sets is a weak uniqueness set.

Proof. Since for sets $E_{1}, E_{2} \in \mathfrak{R}(G)$ the equality $h\left(E_{1} \cup E_{2}\right)=h\left(E_{1}\right) \cap$ $h\left(E_{2}\right)$ holds, our theorem is an immediate consequence of Lemma 4.1.

THEOREM 4.3. Let $G$ be an infinite discrete group for which $k(\mathfrak{T}(G))=\{0\}$. Then every weak uniqueness set in $\Re(G)$ has empty interior.

Proof. Suppose that $E$ is a weak uniqueness set and let $E_{1}$ be the complement of $E$ in $\Re(G)$. Choose an arbitrary function $f$ in $k\left(E_{1}\right)$. Since $k(E) \cap k\left(E_{1}\right)=k\left(E \cup E_{1}\right)=\{0\}$, we see that $f * g=0$ for all $g \in k(E)$. Therefore $f$ annihilates the ideal $k(E)$. But $E$ is a weak uniqueness set, so $f=0$. Thus $k\left(E_{1}\right)=\{0\}$ and so $\bar{E}_{1}=\mathfrak{N}(G)$ which means that $E$ has empty interior.

REMARK. It is known that the class of groups $G$ for which $k(\mathfrak{N}(G))=\{0\}$ (we say then the group algebra $l^{1}(G)$ is strongly semisimple) contains FCgroups [13] and all maximal almost periodic groups [4]. In particular, it contains free groups [17] and finite extensions of abelian groups [6]. No example is known of a discrete group for which $k(\mathscr{T}(G)) \neq\{0\}$.

5. Groups of type $U$. A discrete group $G$ will be called a group of type $U$ if every maximal two-sided ideal in $l^{1}(G)$ is weakly* dense in $l^{1}(G)$. It follows by Theorem 4.2 that $G$ is a group of type $U$ if and only if every finite subset in $\Re(G)$ is a weak uniqueness set. It is clear that finite groups are never of type $U$. If $G$ is an abelian group, then every maximal ideal in $l^{1}(G)$ has codimension one, and Theorem 2.3 implies that infinite abelian groups are of type $U$. We will show that some other classes of groups are also of type $U$.

Proposition 5.1. Let $\mathbb{Q}$ be a complex Banach algebra with a unit and let I be a maximal two-sided ideal in $Q$. Then the center of the algebra $Q / I$ is one-dimensional.

Proof. Let $I_{0}$ be a maximal left ideal in $\mathbb{Q}$ containing $I$. Let $\pi$ denote the left regular representation of $\mathcal{Q}$ in $\mathcal{Q} / I$. It is easy to verify that $\pi$ is an algebraically irreducible representation and ker $\pi=I$. Moreover, the algebras $\mathbb{Q} / I$ and $\pi(\mathscr{Q})$ are isometrically isomorphic. Since $\pi$ is an irreducible representation, the Banach algebra of intertwining operators of $\pi$ is a field 
and by the Gelfand-Mazur Theorem it is isomorphic to the field of complex numbers and since the center of $\mathbb{Q} / I$ intertwines $\pi$, our proposition follows.

COROLlaRY 5.2. Let $\mathbb{Q}$ be a complex Banach algebra with a unit and let $\mathscr{Z}(\mathbb{Q})$ denote the center of $\mathcal{Q}$. If $I$ is a maximal two-sided ideal in $\mathcal{Q}$, then $\mathscr{Z}(\mathbb{Q}) \cap I$ is a maximal ideal in $\mathscr{Z}(\mathbb{Q})$.

Proof. If $\varphi$ denotes the natural homomorphism of $\mathscr{Q}$ onto $\mathscr{Q} / I$, then $\left.\varphi\right|_{\mathscr{L}(\mathbb{Q})}$ is a homomorphism into the field of complex numbers.

LEMMA 5.3. Let $I$ be a left (not necessarily closed) ideal in $l^{1}(G)$. If, for a nonvoid subset $H \subset G$, the set $I \cap l^{1}(H)$ is weakly* dense in $l^{1}(H)$, then $I$ is weakly* dense in $l^{1}(G)$.

Proof. Suppose to the contrary that $I$ is not weakly* dense in $l^{1}(G)$. Then there is a nonzero function $\varphi$ in An $I \cap c_{0}(G)$. Since both An $I$ and $c_{0}(G)$ are invariant under left translations, we may assume (translating $\varphi$ if necessary) that $\left.\varphi\right|_{H} \neq 0$. Hence $\left.\varphi\right|_{H}$ belongs to $c_{0}(H)$ and annihilates $I \cap l^{1}(H)$, but this contradicts the assumption. $U$.

THEOREM 5.4. If a discrete group has infinite center, then it is a group of type

Proof. Let $H$ denote the center of $G$. Since $l^{1}(H) \subset \mathscr{I}\left(l^{1}(G)\right)$, by Corollary 5.2, $I \cap l^{1}(H)$ is a maximal ideal in $l^{1}(H)$. Therefore the theorem follows from Lemma 5.3.

COROLLARY 5.5. Finitely generated infinite nilpotent groups are of type $U$.

Proof. Such groups have infinite centers [15].

REMARKs. (1) There exists an infinite nilpotent group (even of class two) which is not of type $U$ group. An example will be given in $\$ 7$.

(2) The property "to be a group of type $U$ " is neither hereditary nor stable under homomorphisms. Indeed, if $G_{1}$ is an arbitrary group and $G_{2}$ is an infinite abelian group, then $G=G_{1} \times G_{2}$ is a group of type $U$. Observe that although $G_{1}$ is both a subgroup of $G$ and a homomorphic image of $G$, it need not be a group of type $U$.

THEOREM 5.6. Every finite extension of an infinite abelian group is a group of type $U$.

Proof. ${ }^{3}$ Let $H$ be the abelian group whose finite extension is $G$. For $g \in G$ and $h \in H$ denote $h^{g}=g^{-1} h g$. Since elements from the same coset in $G / H$

\footnotetext{
${ }^{3}$ The algebra $l^{\prime}(G)$ is symmetric for such a group [G] and every irreducible unitary representation of $G$ is finite-dimensional [18]. Using these facts one can prove that every maximal two-sided ideal in $l^{1}(G)$ is of finite codimension and thus, by Theorem 2.3, one can get another proof of Theorem 5.6.
} 
define the same automorphisms of $H$, for every $h \in H$ the function $\sum_{\bar{g} \in G / H} h^{\bar{s}}$ belongs to the center of $l^{1}(G)$. By Corollary 5.2 there exists a scalar $\lambda(h)$ such that $\lambda(h) e-\sum_{\bar{g} \in G / H} h^{\bar{g}}$ belongs to $I$. Choose a character $\sigma \in \hat{H}$ from the hull of the ideal $I \cap l^{1}(H)$. Then

$$
\sum_{\bar{g} \in G / H} \sigma_{\bar{g}}(h)=\lambda(h), \quad h \in H,
$$

where $\sigma_{\bar{g}}$ denotes the character of $H$ which at a point $h$ takes value $\sigma\left(h^{g}\right)$. It follows that $\lambda$ is an almost periodic nonzero function on $H$. Suppose now that $\varphi \in \operatorname{An} I \cap c_{0}(G)$. If $\varphi$ is a nonzero function, we may assume that $\varphi(e)=1$. But then

$$
0=\left\langle\lambda(h) e-\sum_{\bar{g} \in G / H} h^{\bar{g}}, \varphi\right\rangle=\lambda(h)-\sum_{\bar{g} \in G / H} \varphi\left(h^{g}\right)
$$

which gives $\lambda \in c_{0}(H)$, impossible because $\lambda$ is an almost periodic function. Therefore An $I \cap c_{0}(G)=\{0\}$ and $I$ is weakly* dense in $l^{1}(G)$.

In the next section we shall give a simple characterization of type $U$ groups among FC groups.

6. FC groups. A discrete group $G$ is said to be an $F C$ group if the orbit of any element of $G$ under the action of all inner automorphisms is finite. Let $G$ be an FC group and let

$$
K_{x}=\left\{y \in G: y=h^{-1} x h \text { for an } h \in G\right\}
$$

denote the orbit of $x$. A bounded complex function $\varphi$ on $G$ is called a character (in the sense of Thoma [19]) if it satisfies the following equation

$$
\varphi(x) \varphi(y)=\frac{1}{\left|K_{x}\right|} \sum_{z \in K_{x}} \varphi(z y) .
$$

Let $E(G)$ denote the topological space of all characters on $G$ satisfying $\varphi(e)=1$, with the topology of pointwise convergence.

Proposition 6.1 (KANiUTh AND Steiner [9]). Let $G$ be an FC group. The map $\varphi \rightarrow M_{\varphi}$, where

$$
M_{\varphi}=\left\{f \in l^{1}(G):\left\langle f * f^{*}, \varphi\right\rangle=0\right\},
$$

is a homeomorphism of $E(G)$ onto $\Re(G)$.

Theorem 6.2. Let $\varphi \in E(G)$. The ideal $M_{\varphi}$ is weakly* dense in $l^{1}(G)$ if and only if $\varphi \notin c_{0}(G)$.

Proof. By the Schwarz inequality it follows at once that $\varphi \in \operatorname{An} M_{\varphi}$. Therefore, if $\varphi$ belongs to $c_{0}(G)$, then $M_{\varphi}$ is not weakly* dense in $l^{1}(G)$. To prove the converse, suppose that there exists a nonzero function $\psi$ in $c_{0}(G)$ 
which annihilates $M_{\varphi}$ (we may assume, as in the proof of Lemma 5.3, that $\psi(e)=1)$. One can see that the function $\psi^{\#}$ defined by

$$
\psi^{\#}(x)=\frac{1}{\left|K_{x}\right|} \sum_{z \in K_{x}} \psi(z)
$$

belongs to $c_{0}(G)$ and $\psi^{\#}(e)=\psi(e)=1$. Let $\mathscr{Z}$ denote the center of the algebra $l^{1}(G)$. It is easy to see that $f^{\#}=f$ for all $f$ in $\mathscr{Z}$. Let $f \in M_{\varphi} \cap \mathscr{Z}$. Then $0=\langle f, \psi\rangle=\left\langle f^{\#}, \psi\right\rangle=\left\langle f, \psi^{\#}\right\rangle$ and therefore $\psi^{\#}$ annihilates $M_{\varphi} \cap$ $\mathscr{Z}$. By Corollary 5.2, $M_{\varphi} \cap \mathcal{Z}$ is a maximal ideal in $\mathscr{Z}$, and so it is of codimension one in $\mathscr{Z}$. Since both $\varphi$ and $\psi^{\#}$ annihilate it, they are linearly dependent as functionals on $\mathscr{Z}$. Thus there exists a complex number $\lambda$ such that $\left\langle f, \varphi-\lambda \psi^{\#}\right\rangle=0$ for all $f$ in $\mathscr{Z}$. Taking first $f=\delta_{e}$, we get $\lambda=1$, and taking $f=\delta_{x}^{\#}$, where $x$ is an arbitrary element in $G$, we get $0=\left\langle\delta_{x}^{\#}, \varphi-\right.$ $\left.\psi^{\#}\right\rangle=\left\langle\delta_{x}, \varphi-\psi^{\#}\right\rangle=\varphi(x)-\psi^{\#}(x)$. Therefore $\varphi=\psi^{\#}$, whence $\varphi \in$ $c_{0}(G)$, and out theorem follows.

Corollary 6.3. An FC group $G$ is a type $U$ group if and only if $E(G) \cap$ $c_{0}(G)=\varnothing$.

7. Examples. To illustrate our previous considerations we will now give some examples, which also seem interesting in themselves. In the first one we investigate an FC group $G$, which is also a nilpotent group of class two. For that group the space $\Re(G)$ is Hausdorff and possesses exactly one isolated point. Consequently the maximal two-sided ideal in $l^{1}(G)$ corresponding to it is not weakly* dense in $l^{1}(G)$ and therefore $G$ is not a group of type $U$. The second example is concerned with the infinite dihedral group. Here the space $\mathfrak{N}(G)$ is connected but not Hausdorff, and the group is of type $U$. In the last example we build a group $G$ for which $\Re(G)$ has a dense subset of isolated points. Of course, such a group cannot be of type $U$. It is interesting that $l^{1}(G)$ is strongly semisimple while $G$ is neither an FC group nor an MAP group.

EXAMPLE 1. Let $G$ be an infinite group for which the center $\mathscr{Z}(G)$ is equal to the commutator group $G^{\prime}$ and consists of two elements. ${ }^{4}$ Let us denote these elements by $e$ and $u$. It is clear that $K_{e}=\{e\}, K_{u}=\{u\}$ and $K_{x}=$ $\{x, u x\}$ for $x \notin \mathscr{Z}(G)$. Hence $G$ is an FC group and to describe the space $\mathfrak{N}(G)$ it suffices to find all the characters in $E(G)$.

4 The existence of such a group was communicated to us by Professor E. Kaniuth. In the construction we follow [10].

Let $K$ be a noncommutative eight-element group (here $\mathscr{Z}(K)=K^{\prime}$ and has two elements). Let $G_{0}=\bigoplus_{n=1}^{\infty} K_{n}$, where $K_{n}=K$ for all $n$. Since $\mathscr{Z}\left(G_{0}\right)=\bigoplus_{n=1}^{\infty} \mathscr{L}\left(K_{n}\right)$, the mapping $\varphi\left(\left\{x_{n}\right\}_{n=1}^{\infty}\right)$ $=x_{1} x_{2} \cdots$ is a homomorphism from $\mathscr{L}\left(G_{0}\right)$ onto $\mathscr{I}(K)$. The group $H=\operatorname{ker} \varphi$ is a subgroup of $\mathscr{Z}\left(G_{0}\right)$ and so it is a normal subgroup in $G_{0}$. The quotient group $G_{0} / H$ has the required properties. 
Suppose $\varphi \in E(G)$. Then $\left.\varphi\right|_{\mathscr{L}(G)} \in \mathscr{Z}(G)^{\wedge}$ and we have two possibilities: either $\varphi(u)=-1$ or $\varphi(u)=1$. In the first case the equation (*) in $\S 6$ yields $\varphi(x)=0$ for $x \notin \mathscr{Z}(G)$. Let us denote this character by $\varphi_{0}$. In the second case $\varphi$ is constant on cosets with respect to $\mathscr{Z}(G)=G^{\prime}$ and hence $\varphi$ is a character of the abelian group $G / G^{\prime}$. This shows that the space $\Re(G)$ is homeomorphic with the space $\left(G / G^{\prime}\right)^{\wedge} \cup\left\{\varphi_{0}\right\}$, in which $\varphi_{0}$ is an isolated point and the topology in $\left(G / G^{\prime}\right)^{\wedge}$ is the ordinary Pontriagin topology. Observe that $\varphi_{0}$ belongs to $c_{0}(G)$ and so the maximal two-sided ideal corresponding to it is not weakly* dense in $l^{1}(G)$. Therefore $G$ is not a group of type $U$.

Groups presented in Examples 2 and 3 below are semidirect products of abelian groups. For such groups the description of the space $\mathfrak{N}(G)$ was given in [14] and runs as follows.

Let $G$ be a semidirect product of two abelian subgroups $K$ and $N$, where $N$ is a normal subgroup and $K$ acts on $N$. Then $K$ acts in a natural way on the dual group $\hat{N}$. Let us denote by $\sigma_{k}$ the image of $\sigma \in \hat{N}$ under the action of $k \in K$ and let $K_{\sigma}$ denote the stability group of $\sigma$. For $(\tau, \sigma) \in \hat{K} \times \hat{N}$ the set

$$
O_{(\tau, \sigma)}=\left\{\left(\tau \chi, \sigma_{k}\right) \in \hat{K} \times \hat{N}: \chi \in\left(K / K_{\sigma}\right)^{\wedge}, k \in K / K_{\sigma}\right\}
$$

will be called the orbit of the point $(\tau, \sigma)$. The set of all orbits determines an equivalence relation $\sim$ in $\hat{K} \times \hat{N}$. In the particular case when all orbits are closed, the space $\mathfrak{T}(G)$ is homeomorphic to the topological space $\hat{K} \times$ $\hat{N} / \sim$. The maximal two-sided ideal in $l^{1}(G)$ corresponding to an orbit $O_{(\tau, \sigma)}$ consists exactly of those summable functions on $K \times N$ whose ordinary Fourier transforms vanish on $O_{(\tau, \sigma)}$.

EXAMPLE 2. Let $G$ be the infinite dihedral group. It is a free group on two generators $a, b$ with relations $a^{2}=b^{2}=e$. Since the cyclic subgroup generated by $a b$ is a normal subgroup of index two in $G$, the group $G$ is isomorphic to a semidirect product of two abelian subgroups $K$ and $N$, where $K$ is the two-element group $\{-1,1\}$ and $N$ is the group of integers. The action of $K$ on $N$ is the ordinary multiplication. If we identify the group $\hat{K} \times \hat{N}$ with $\{-1,1\} \times T$, where $T$ denotes the circle group, then the orbits $O_{(\tau, \sigma)}$ are of the following form: $O_{(\tau, \sigma)}=\{(\tau, \sigma)\}$ if $\sigma=1$ or $\sigma=-1$ and $O_{(\tau, \sigma)}=$ $\{(-1, \sigma),(1, \sigma),(-1, \bar{\sigma}),(1, \bar{\sigma})\}$ if $\sigma \neq \pm 1$. One can observe that the space भR $(G)$ being homeomorphic to $\hat{K} \times \hat{N} / \sim$ can be realized as the "symmetric four-point compactification" of the open interval $(0, \pi)$ by adding two points to each end of the interval. Therefore $\Re(G)$ is a connected topological space, but not Hausdorff. The group $G$ considered here is a finite extension of an infinite abelian group, and so by Theorem 5.6 it is a type $U$ group.

EXAMPLE 3. Let $G_{1}=Z\left(2^{\infty}\right)$ be the multiplicative discrete group of all numbers $\exp \left(2 \pi i k / 2^{n}\right)$, where $k$ runs through all integers and $n$ runs through 
all nonnegative integers, and let $G_{2}$ be the group of all automorphisms of $G_{1}$. We would like to describe the space $\Re(G)$ where $G$ is a semidirect product of $G_{2}$ and $G_{1}$ where elements of the group $G_{2}$ act on $G_{1}$ as automorphisms.

It is convenient to consider the group $G_{1}$ as a direct limit of groups $A_{n}$ of all $2 n$th roots of unity with the natural embeddings

$$
A_{1} \rightarrow A_{2} \rightarrow \cdots \rightarrow A_{n} \rightarrow A_{n+1} \rightarrow \cdots \text {. }
$$

It follows that the character group $\hat{G}_{1}$ of $G_{1}$ is isomorphic to the inverse limit of $A_{n}$ 's,

$$
\cdots \rightarrow A_{n+1} \rightarrow A_{n} \rightarrow \cdots \rightarrow A_{2} \rightarrow A_{1} \text {, }
$$

that is to say to the additive group $\left(Z_{2},+\right)$ of the ring $Z_{2}$ of 2 -adic numbers. One can observe that the group $G_{2}$ is algebraically isomorphic to the multiplicative group $Z_{2}^{*}$ of units of the ring $Z_{2}$ and the action of $G_{2}$ on $\hat{G}_{1}$ corresponds to the multiplication in the ring $Z_{2}$. Now we would like to describe the forms of the orbits in $\hat{G}_{2} \times\left(Z_{2},+\right)$. Fix $(\tau, x) \in \hat{G}_{2} \times\left(Z_{2},+\right)$. If $x=0$, then the stability group $K_{0}$ equals $G_{2}$, and thus $O_{(\tau, 0)}=\{(\tau, 0)\}$. If $x \neq 0$, then it is of the form $x=2^{n-1} u$ for some $n \in N$ and $u \in Z_{2}^{*}$. Since the ring $Z_{2}$ has no zero divisors, the stability group $K_{x}$ is trivial and an easy argument shows that the orbit $O_{(\tau, x)}$ is of the form

$$
O_{(\tau, x)}=\hat{G}_{2} \times 2^{n-1} Z_{2}^{*}
$$

and hence it is an open-closed subset in $\hat{G}_{2} \times\left(Z_{2},+\right)$. Since all orbits are closed, the space $\Re(G)$ is homeomorphic to the " $\hat{G}_{2}$ compactification of the natural numbers $N$ ". More precisely, $\Re(G)=N \cup \hat{G}_{2}$ as a set. A set $U$ is open in $N \cup \hat{G}_{2}$ if either $U \subset N$ or $U \cap \hat{G}_{2}$ is a nontrivial open set in $\hat{G}_{2}$ and $N-U$ is finite. Observe that the set $N$ consists of isolated points and it is dense in $\mathscr{R}(G)$.

The group algebra $l^{1}(G)$ is strongly semisimple. Indeed, if a function $f$ belongs to every maximal two-sided ideal, then its Fourier transform $\hat{f}$ is zero on each orbit $O_{(\tau, x)}$. Thus $\hat{f}$ is zero everywhere on $\hat{G}_{2} \times\left(Z_{2},+\right)$ and so $f$ is zero too.

Since the space $\Re(G)$ has isolated points, the group $G$ is not maximally almost periodic. ${ }^{5}$ Moreover, by Theorem 4.3, a maximal two-sided ideal corresponding to such a point is not weakly* dense in $l^{1}(G)$. Hence $G$ is not a group of type $U$.

${ }^{5}$ Let $\pi$ be a finite-dimensional irreducible representation of $G$. Then ker $\pi$ is a maximal two-sided ideal in $l^{1}(G)$ and is weakly* dense there (has finite codimension). Let $G$ be a MAP group. If $\Re(G)$ has an isolated point then, by Theorem 4.3, it is not weakly* dense in $l^{1}(G)$ and so it is not the kernel of a finite-dimensional representation. Let $I=k(\Re(G)-\{M\})$. Then $I$ is a nontrivial subset of $l^{1}(G)$, contained in every maximal two-sided ideal excepting $M$. In particular, it is annihilated by every finite-dimensional irreducible representation. But this contradicts the assumption that $G$ is a MAP group. 


\section{REFERENCES}

1. N. Bary, A treatise on trigonometric series, vols. 1, 2 (English translation), Pergamon Press, Oxford, 1964.

2. J. Dixmier, Les $C^{*}$-algèbres et leurs représentations, Gauthier-Villars, Paris, 1964.

3. R. Godement, Mémoire sur la théorie des caractères dans les groupes localement compacts unimodulaires, J. Math. Pures Appl. 30 (1951), 1-110.

4. S. Grosser and M. Moskowitz, Harmonic analysis on central topological groups, Trans. Amer. Math. Soc. 156 (1971), 419-454.

5. E. Hewitt and K. A. Ross, Abstract harmonic analysis, vols. 1, 2, Springer, Berlin, $1963 / 1970$.

6. A. Hulanicki, On symmetry of group algebras of discrete nilpotent groups, Studia Math. 35 (1970), 207-219.

7. On the spectral radius of hermitian elements in group algebras, Pacific J. Math. 18 (1966), 277-287.

8. E. Kaniuth and G. Schlichting, Zur harmonischen Analyse klassenkompaktten Gruppen II, Inventiones Math. 10 (1970), 332-345.

9. E. Kaniuth and D. Steiner, On complete regularity of group algebras, Math. Ann. 204 (1973), 305-329.

10. I. Kaplansky, Group algebras in the large, Tôhoku Math. J. 3 (1951), 249-256.

11. T. W. Körner, A pseudo function on a Helson set, Astérisque, 5, S.M.F., 1973.

12. Y. Meyer, Algebraic numbers and harmonic analysis, North-Holland, Amsterdam, 1970.

13. R. D. Mosak, The $L^{1}$ - and $C^{*}$-algebras of $[F I A]_{B}$ groups and their representations, Trans. Amer. Math. Soc. 163 (1972), 277-310.

14. T. Pytlik, Harmonic analysis on semi-direct product of abelian groups, J. Functional Analysis (submitted).

15. Derek J. S. Robinson, Finiteness conditions and generalized soluble group. I, II, SpringerVerlag, Berlin and New York, 1972.

16. W. Rudin, Fourier analysis on groups, Interscience, New York, 1962.

17. I. E. Segal, The group algebra of a locally compact group, Trans. Amer. Math. Soc. 61 (1947), 69-105.

18. E. Thoma, Über unitäre Darstellungen ab zählbarer diskreten Gruppen, Math. Ann. 163 (1964), 111-138.

19. $20-42$.

, Zur harmonischen Analyse klassenfiniter Gruppen, Inventiones Math. 3 (1967),

20. A. Zygmund, Trigonometric series, 2nd ed., vol. 2, Cambridge University Press, Cambridge, 1959.

Institute of Mathematics of the Polish Academy of Sciences, Wroceaw, Poland

Institute of Mathematics, University of Wrockaw, Wrockaw, Poland 